\title{
Parameters influencing high-efficiency transfection of bacterial artificial chromosomes into cultured mammalian cells
}

\author{
William J. Montigny, Stephanie F. Phelps, Sharon Illenye, and Nicholas H. Heintz
}

BioTechniques 35:796-807 (October 2003)

\begin{abstract}
Although bacterial artificial chromosomes (BACs) provide a well-characterized resource for the analysis of large chromosomal domains, low transfection rates have proven a significant limitation for their use in cell culture models. Using TP53 BAC clones that contain expression cassettes for enhanced green fluorescent protein or red fluorescent protein, we have examined conditions that promote BAC transfection in hamster, human, and mouse cell lines. Atomic force microscopy shows that BAC transfection efficiency correlates with the generation of small, highly condensed but dispersed lipid:BAC DNA transfection complexes. BAC DNA purity and concentration are critical for good transfection; debris from purification columns induces the formation of large aggregates that do not gain entry into the cell, and DNA concentrations must be optimized to promote intramolecular condensation rather than intermolecular linking, which also causes aggregation and diminished transfection efficiency. The expression of both markers and genes within BACs initially occurs at lower levels than observed with plasmids, requiring 3-5 days to evaluate the transfection results. We also show that BACs can be co-transfected with other BACs, which provides for increased experimental flexibility.
\end{abstract}

\section{INTRODUCTION}

Bacterial artificial chromosomes (BACs), P1-derived artificial chromosomes (PACs), and yeast artificial chromosomes (YACs) provide a resource for the functional analysis of large blocks of genomic sequence, including entire genes, gene families, replicons, or other chromosomal domains. For example, transgenic mice generated with modified BACs have proven valuable for studying cis-acting elements that act at a distance to regulate tissuespecific and developmental patterns of gene expression (1-4). Because of their stability and the fact that they have been used to map and sequence the human and mouse genomes, BACs currently represent the most completely characterized source of large genomic fragments. Moreover, several methods for using homologous recombination in bacteria to introduce specific sequence alterations in BACs have been described (5-8), thereby permitting the same types of genetic manipulations that have long been available in plasmid-based systems.

Lipid-based delivery systems have proven extremely effective for achieving high rates of plasmid transfection in mammalian cells $(9,10)$. Commercially available preparations have been highly refined and consist of a cationic polymer or amphiphile, sometimes in combination with a neutrally charged fusogenic lipid $(10,11)$. The cationic nature of the lipid enables it to form a complex with DNA, probably through interactions with the negatively charged phosphate backbone, forming multilamellar micelle-like structures containing multiple DNA molecules (12). Although the processes that lead to efficient in vitro transfection remain unclear, it is evident that the pathway involves initial DNA-lipid complex formation, followed by fusion with the membrane and entry of the lipoplex into the cell cytoplasm (13). Finally, the DNA must be released from the lipid complex and gain entry into the nucleus, where it will be accessible to the transcription machinery (14-16).
We are interested in the interaction of regulatory elements at a distance that controls long-range chromatin organization, gene expression, and DNA replication. Because the generation of transgenic mice is expensive and timeconsuming, we investigated methods for the use of BACs for routine transfection assays in cell culture models. Here we describe several parameters that influence high-efficiency transfection of BACs into cultured cells.

\section{MATERIALS AND METHODS}

\section{Modification of TP53 BAC Clones}

To track BAC transfection, an expression cassette for enhanced green fluorescent protein (EGFP) was introduced into the pBeloBAC11 vector backbone of a BAC that contained the entire human TP53 tumor suppressor gene (17), as previously described (5). The BAC modification vector pLD55A-CMV-EGFP-B-recA was derived 
from pLD55 (generous gift from Barry L. Wanner, Purdue University, West Lafayette, IN, USA) and contained the 1.4-kb Escherichia coli recA gene, a 1.6-kb CMV-EGFP-pA expression cassette from pEGFP-N1 (BD Biosciences Clontech, Palo Alto, CA, USA), and two homology arms (Figure 1, A and $\mathrm{B} ; 0.4$ and $0.48 \mathrm{~kb}$, respectively) from the pBeloBAC11 vector (GenBank $^{\circledR}$ accession no. U51113) to target the homologous recombination event. The 11.4-kb pLD55-A-CMVEGFP-B-recA vector was mapped and sequenced to confirm its organization. Cells containing the human TP53 BAC $66 \mathrm{H} 22$ were made electrocompetent and transformed with $1 \mu \mathrm{g}$ pLD55-ACMV-EGFP-B-recA modification vector. The cells were allowed to recover for $60 \mathrm{~min}$ and then were plated on LB plates containing $12.5 \mu \mathrm{g} / \mathrm{mL}$ chloramphenicol (Chl) and $100 \mu \mathrm{g} / \mathrm{mL}$ ampicillin (Amp) and incubated overnight at $37^{\circ} \mathrm{C}$. Minipreparations of BAC DNA were prepared as previously described [BACPAC Resources, Children's Hospital Oakland Research Institute (http://
A

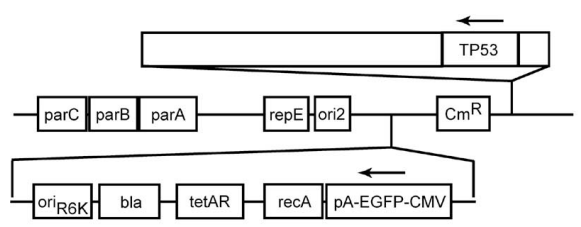

C

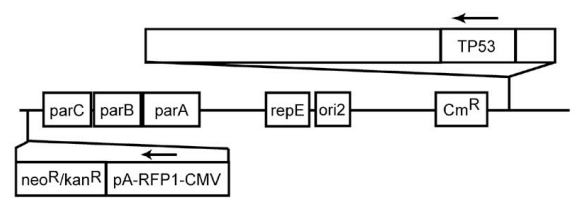

D

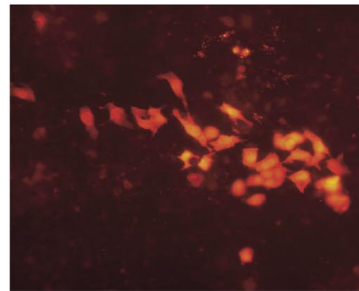

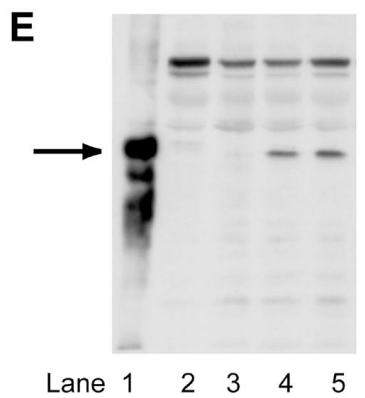

Figure 1. Monitoring bacterial artificial chromosome (BAC) transfection with TP53 BACs containing enhanced green fluorescent protein (EGFP) and red fluorescent protein (RFP) expression cassettes. (A) Human TP53 BAC 66H22 was modified by homologous recombination (5) to contain a cytomegalovirus (CMV)-EGFP expression cassette in the pBeloBAC11 vector backbone, which resulted in BAC clone B16-25. (B) Fluorescence microscopy showed the transfection of B16-25 into CHOC 400 cells using a modified protocol with PLUS reagent and Lipofectamine resulted in EGFP-positive cells by $72 \mathrm{~h}$. (C) Using ET cloning (6), a CMV-RFP expression cassette was introduced into the pBeloBAC11 vector of 265A8 to generate BAC N2A1. (D) The transfection of N2A1 into mouse C10 cells using Lipofectamine 2000 resulted in RFP-positive cells by $72 \mathrm{~h}$. (E) Extracts were prepared $72 \mathrm{~h}$ after the transfection of CHOC 400 cells with B16-25, (lane 3) without and (lanes 4-5) with an 8-h treatment with $25 \mu \mathrm{M}$ cis-platinum to stabilize $\mathrm{p} 53$. The extracts were examined for p53 expression by immunoblotting anti-human p53 antibody Ab-3. Lane 1, control extract from cells transfected with a pCMV-p53 expression plasmid. Lane 2, sham transfection control. Previous studies showed that CHOC 400 cells do not express p53 (17). 
bacpac.chori.org/bacpacmini.htm)], and final pellets were resuspended in $25 \mu \mathrm{L}$ TE $(10 \mathrm{mM}$ Tris-HCl, pH 8.0, $1 \mathrm{mM}$ EDTA). A portion of each BAC DNA miniprep $(15 \mu \mathrm{L})$ was analyzed by digestion with restriction endonucleases and Southern blot analysis for the integration of the modification vector into the pBeloBAC11 vector backbone. B16-25 was recovered as a stable co-integrant and propagated for DNA preparation in $12.5 \mu \mathrm{g} / \mathrm{mL} \mathrm{LB} /$ Chl per $100 \mu \mathrm{g} / \mathrm{mL}$ Amp.

For co-transfection studies, TP53 BAC 265A8 was modified to contain a CMV-RFP-kanR expression cassette using recE and recT to mediate homologous recombination (ET cloning), as previously described (6). A 251-bp fragment from pBeloBAC11 (bases 6841-7092) was amplified by PCR and cloned into pBlueScript ${ }^{\mathrm{TM}}$ (Stratagene, La Jolla, CA, USA). The vector was cut within the 251-bp insert with BstEII, and a 3.6-kb BstEII fragment containing CMV-RFP-kanR (pDsRed-N1; BD
Biosciences Clontech) was inserted by ligation, thereby providing sequences homologous to pBeloBAC11 flanking the red fluorescent protein (RFP) expression cassette. The BstEII fragment containing CMV-RFP-kanR and targeting sequences was amplified by PCR, digested with DpnI to remove template DNA, and $0.3 \mu \mathrm{g}$ DNA was used to transform into cells harboring BAC 265A8 and pBAD- $\alpha \beta \gamma$ (generous gift from A. Francis Stewart, Technical University, Dresden, Germany) by electroporation. The induction of recombination proteins from pBAD$\alpha \beta \gamma$ in the electrocompetent cells was accomplished by the addition of arabinose to $0.1 \% 1 \mathrm{~h}$ before harvest. Transformed cells were plated onto LB plates containing $12.5 \mu \mathrm{g} / \mathrm{mL} \mathrm{Chl}$ and $20 \mu \mathrm{g} / \mathrm{mL}$ kanamycin (Kan). Minipreparations of BAC DNA from the resulting transformants were analyzed for the introduction of the RFP marker by digestion with restriction endonucleases and Southern blot analysis as described earlier. The resulting 265A8 BAC clone that had CMV-RFP-kanR stably integrated was called N2A1, and it was propagated in $12.5 \mu \mathrm{g} / \mathrm{mL}$ $\mathrm{LB} / \mathrm{Chl}$ per $20 \mu \mathrm{g} / \mathrm{mL}$ Kan.

\section{Large-Scale Preparation of BAC DNA}

BAC DNA was prepared by several methods, including standard preparation using equilibrium sedimentation in cesium chloride (5). Several commercial preparation methods were employed as follows. For each method, a single colony was inoculated into $250 \mathrm{~mL} \mathrm{LB} / \mathrm{Chl}(12.5 \mu \mathrm{g} / \mathrm{mL})$ in triple baffled Erlemeyer shake flasks (Bellco Glass, Vineland, NJ, USA) and grown to saturation overnight at $37^{\circ} \mathrm{C}$ with shaking at $225 \mathrm{rpm}$. For the Concert ${ }^{\mathrm{TM}}$ (Marligen, Ijamsville, MD, USA), Nucleobond ${ }^{\mathrm{TM}}$ (BD Biosciences Clontech), and Qiagen (Stanford, CA, USA) Kits, BAC DNA was isolated according to each manufacturer's protocol without modification. In all instances, the final BAC DNA pellet was air-dried for $10 \mathrm{~min}$, resuspended in $100-300 \mu \mathrm{L}$ $\mathrm{TE}$, heated for $10 \mathrm{~min}$ at $65^{\circ} \mathrm{C}$, and allowed to resuspend from overnight to several days at $4^{\circ} \mathrm{C}$. The concentration was then determined by absorbance spectrophotometry at 260 and $280 \mathrm{~nm}$, and the samples were diluted in TE to a final concentration of $200-500 \mathrm{ng} / \mu \mathrm{L}$.

\section{Atomic Force Microscopy of BAC DNA and Lipoplexes}

Atomic force microscopy (AFM) studies were performed using a BioScope $^{\mathrm{TM}}$ Atomic Force Microscope (Digital Instruments, Santa Barbara, CA, USA) equipped with a Plexiglas ${ }^{\circledR}$ tapping mode fluid cell. The AFM was operated in fluid-tapping mode using cantilever frequencies between 12 and $24 \mathrm{kHz}(18,19)$. Triangular silicon nitride cantilevers $(100 \mu \mathrm{m})$ with oxidesharpened oriented tips with a normal spring constant of $0.38 \mathrm{~N} / \mathrm{m}$ were used (Advanced Surfaces Microscopy, Indianapolis, IN, USA). Plasmid and BAC DNA was diluted to a final concentration of $1-5 \mathrm{ng} / \mathrm{\mu L}$ in imaging buffer $(20 \mathrm{mM}$ Tris- $\mathrm{HCl}$, pH 7.5, $5 \mathrm{mM} \mathrm{MgCl}_{2}, 5 \mathrm{mM}$ $\mathrm{KCl}, 1 \mathrm{mM} \beta$-mercaptoethanol, $2 \mathrm{mM}$ $\mathrm{ZnCl}_{2}$ ), and $7-10 \mu \mathrm{L}$ were deposited on freshly cleaved mica discs affixed to glass slides with epoxy (20). After 2-5 min at room temperature, $200 \mu \mathrm{L}$ imaging buffer were deposited on the mica, and the samples were imaged under ambient fluid conditions $(18,20)$. The images were analyzed using Nanoscope ${ }^{\mathrm{TM}}$ IIIa Software (Digital Instruments).

BAC and plasmid DNA-lipid complexes (lipoplexes) were prepared using several commercial reagents. Here we present transfection and AFM results for PLUS ${ }^{\mathrm{TM}}$ Reagent, Lipofectamine $^{\mathrm{TM}}$, and Lipofectamine 2000 (Invitrogen, Carlsbad, CA, USA) with either plasmid or BAC DNA. Suggested protocols were modified and optimized for each reagent to reflect the empirical results from the transfections and specific requirements of the AFM instrumentation. All lipoplex formation was done in the imaging buffer at room temperature, which has been shown to allow for the formation of lipid-DNA transfection complexes (17). To achieve images of the early and middle-stage formation of particles when both DNA and lipid may be clearly viewed, the ratio of lipid to DNA was decreased. For BAC DNA-lipid complexes formed using the PLUS reagent, $200 \mathrm{ng}$ BAC DNA were incubated in a volume of 50 $\mu \mathrm{L}$ imaging buffer with $0.5 \mu \mathrm{L}$ PLUS reagent for $30 \mathrm{~min}$ at room tempera- 
ture prior to imaging. For complexes formed using Lipofectamine, $200 \mathrm{ng}$ BAC DNA were incubated in a volume of $50 \mu \mathrm{L}$ imaging buffer with 0.1 $\mu \mathrm{L}$ Lipofectamine for $30 \mathrm{~min}$ at room temperature prior to imaging. For complexes formed using Lipofectamine 2000, 400 ng BAC DNA were incubated for $5 \mathrm{~min}$ in $25 \mu \mathrm{L}$ imaging buffer at room temperature; concomitant with this incubation, $0.3 \mu \mathrm{L}$ Lipofectamine 2000 was incubated in $50 \mu \mathrm{L}$ imaging buffer for $5 \mathrm{~min}$ at room temperature. These two solutions were combined and incubated for $1-2 \mathrm{~h}$ before imaging. A $10-\mu \mathrm{L}$ aliquot of each reaction mixture was then deposited directly onto freshly cleaved mica (or diluted first to $1-5 \mathrm{ng} / \mu \mathrm{L} \mathrm{DNA}$, if needed) and imaged by AFM as described earlier.

\section{Cell Transfection}

CHOC 400 cells (21) and mouse alveolar epithelial type II C10 cells (22) were transfected with plasmid DNA using standard protocols for Lipofectamine, Lipofectamine and PLUS reagent, or Lipofectamine 2000. For BAC transfections, control experiments utilized GST-Z2, a glutathioneS-transferase (GST) fusion protein containing three zinc fingers and the proline-rich region of RIP60 (23), and Lipofectamine as previously described (17). To test different transfection conditions of the CHOC 400 or $\mathrm{C} 10$ cells with BAC B16-25 or BAC N2A1, the cells were plated on glass coverslips in 35-mm dishes in Dulbecco's modified Eagle's medium (DMEM; Invitrogen) with $10 \%$ fetal bovine serum (FBS; Hyclone, Logan, UT, USA) under standard cell culture conditions $\left(37^{\circ} \mathrm{C}\right.$ and $5 \% \mathrm{CO}_{2}$ ) such that they were $50 \%-80 \%$ confluent on the following day. The optimal cell density depended on the nature of the transfection reagent and the length of time after transfection required for cell proliferation. For transfections with Lipofectamine and PLUS reagent, BAC DNA ranging $0.2-6 \mu \mathrm{g}$ per reaction was suspended in DMEM without FBS, 6-15 $\mu$ L PLUS reagent were added to a final volume of $100 \mu \mathrm{L}$, and the reaction was incubated for $15 \mathrm{~min}$ at room temperature. During the incubation, $4-12 \mu \mathrm{L}$ Lipofectamine were diluted in $100 \mu \mathrm{L}$ DMEM in a separate tube. The diluted Lipofectamine was then gently mixed with the DNA:PLUS reagent reaction and incubated at room temperature for an additional $15 \mathrm{~min}$. During the second 15-min incubation, the medium on the 35-mm cell culture plates was replaced with $1 \mathrm{~mL}$ serum-free DMEM, the transfection mixture was then added to the cells, and the lipoplexes were allowed to attach for 5-8 h. At the end of the incubation, the transfection media were completely replaced by DMEM supplemented with $10 \%$ FBS, with or without antibiotics. The cells were then cultured for 1-5 days without replacing the culture medium. Transfections for preparing extracts for immunoblotting for p53 or EGFP were performed using the same protocol, without the use of coverslips.

\section{Transfection of Saos-2 with B16-25}

The transfection conditions for the CHOC 400 and $\mathrm{C} 10$ cells were unsuitable for human osteosarcoma Saos- 2 cells. To refine the transfection conditions for the Saos- 2 cells, the cells were plated on glass coverslips in a 12 -well dish at $3 \times 10^{5}$ cells/well in DMEM with $10 \%$ FBS. The following day, 1.8 or $3 \mu \mathrm{g}$ BAC DNA were diluted in room-temperature OptiMEM $^{\circledR}$ (Invitrogen) to a final volume of $100 \mu \mathrm{L}$. Varying concentrations of Lipofectamine 2000 were diluted with Opti-MEM to a final volume of 100 $\mu \mathrm{L}$ to achieve a ratio of DNA weight to Lipofectamine volume that ranged from 1:1 to 1:3. The Lipofectamine and BAC DNA preparations in Opti-MEM were gently mixed and incubated for $20 \mathrm{~min}$ at room temperature. During this incubation, growth medium was replaced with $1 \mathrm{~mL}$ serum-free DMEM. Transfection complexes were then added to the cell cultures, and the cultures were incubated at $37^{\circ} \mathrm{C}$ in $5 \%$ $\mathrm{CO}_{2}$ as described earlier. After $4 \mathrm{~h}$, the transfection medium was replaced with DMEM and 10\% FBS with antibiotics, and the cells were maintained under standard conditions until fixation.

\section{Cell Imaging Fluorescence Microscopy}

After transfection, the cells on the 
coverslips were rinsed twice in phosphate-buffered saline (PBS), fixed in PBS containing $4 \%$ paraformaldehyde for $15 \mathrm{~min}$, rinsed in PBS, and mounted with VECTASHIELD ${ }^{\circledR}$ (Vector Laboratories, Burlingame, CA, USA). In some instances, nuclei were visualized by staining with $4^{\prime}, 6^{\prime}$-diamidino-2phenylindole (DAPI). Fluorescence microscopy was performed using a BX50 Microscope (Olympus America, Melville, NY, USA).

\section{Immunoblotting}

The cells were rinsed in cold PBS, scraped into $100 \mu \mathrm{L} 2 \times$ sodium dodecyl sulfate (SDS) lysis buffer [4\% SDS, 20\% glycerol, $20 \mathrm{mM}$ dithiothreitol (DTT), $120 \mathrm{mM}$ Tris-HCl, pH.6.8, $0.002 \%$ bromophenol blue], heated to $95^{\circ} \mathrm{C}$ for $10 \mathrm{~min}$, and the samples (approximately $20 \mu \mathrm{g} / \mathrm{lane}$ ) were resolved on $8 \%$ SDS denaturing polyacrylamide gels. After transfer to Immobilon ${ }^{\mathrm{TM}}$ Membranes (Millipore, Bedford, MA, USA), the immunoblots were incubated with primary anti-p53 antibody Ab-3 (Oncogene Sciences, Boston, MA, USA), secondary antibody, and washed as previously described (24). Signals were detected with X-ray film using the Western Lightening $\mathrm{Kit}^{\mathrm{TM}}$ (PerkinElmer Life Sciences, Boston, MA, USA).

\section{RESULTS}

\section{Generation of BACs Marked with EGFP and RFP}

To track the transfection of BAC DNA, we constructed human TP53 BAC clones marked with either EGFP or RFP expression cassettes (Figure 1, A and C). The TP53-CMV-EGFP BAC (B16-25) is approximately 116 $\mathrm{kb}$, whereas the CMV-RFP-kanR TP53 BAC (N2A1) is approximately $110 \mathrm{~kb}$. The transfection of $\mathrm{B} 16-25$ into the CHOC 400 cells resulted in EGFPpositive cells in 24-72 h (Figure 1B). Similar results are obtained when the N2A1 BAC expressing RFP is used for the transfection of mouse $\mathrm{C} 10$ cells (Figure 1D). As in previous experiments that showed that the parental $66 \mathrm{H} 22$ TP53 BAC contains the entire wild-type TP53 gene and expresses p53 from native promoter elements (17), immunoblotting showed that B16-25 also expresses p53 (Figure 1E, lanes 4 and 5). We have no evidence that the introduction of the CMV-EGFP or CMV-RFP-kanR expression cassette into the pBeloBAC11 vector interferes with or augments the expression of p53 (data not shown).

\section{Atomic Force Microscopy of Transfection Components}

An examination of the structure-function relationship of lipoplex transfection particles provides insight into parameters (e.g., particle size and stability) that play integral roles in transfection efficiency. Many biophysical methods, including transmission and cryo-electron microscopy $(25,26), X$-ray diffraction $(27,28)$, and AFM $(29,30)$, have been utilized to examine the nature of DNA-lipid complexes. By utilizing a variation of AFM known as fluid-tapping mode that allows for the examination of biological samples under ambient buffer, we have obtained high-resolution images of DNA-lipid complexes under condi- tions that are nearly identical to those that exist in actual in vitro transfection reactions $(18,19,31)$.

To refine the transfection conditions, we first examined both plasmid and BAC DNA preparations by AFM. Based on appearance, supercoiled, circular, and linear forms of plasmid DNA could be distinguished clearly by AFM (Figure 2A). In contrast to high copy number plasmids, BACs are propagated at 1-2 copies per cell (32), and the purity of DNA preparations as assessed by AFM varied widely. BAC DNA prepared using $\mathrm{CsCl}$ equilibrium gradient sedimentation was uniformly devoid of large debris (Figure 2B). In contrast, BAC DNA prepared using several maxi plasmid or BAC DNA purification kits invariably showed contamination with debris (Figure 2, C-E), which in most instances could be attributed to the dissociation of the resin from the purification columns. In some cases, the debris could be removed by organic extraction with buffer-saturated phenol:chloroform $[24: 1(\mathrm{v} / \mathrm{v})]$ and precipitation with ethanol, but this often resulted in the loss or breakage of the BAC DNA (Figure 2F).
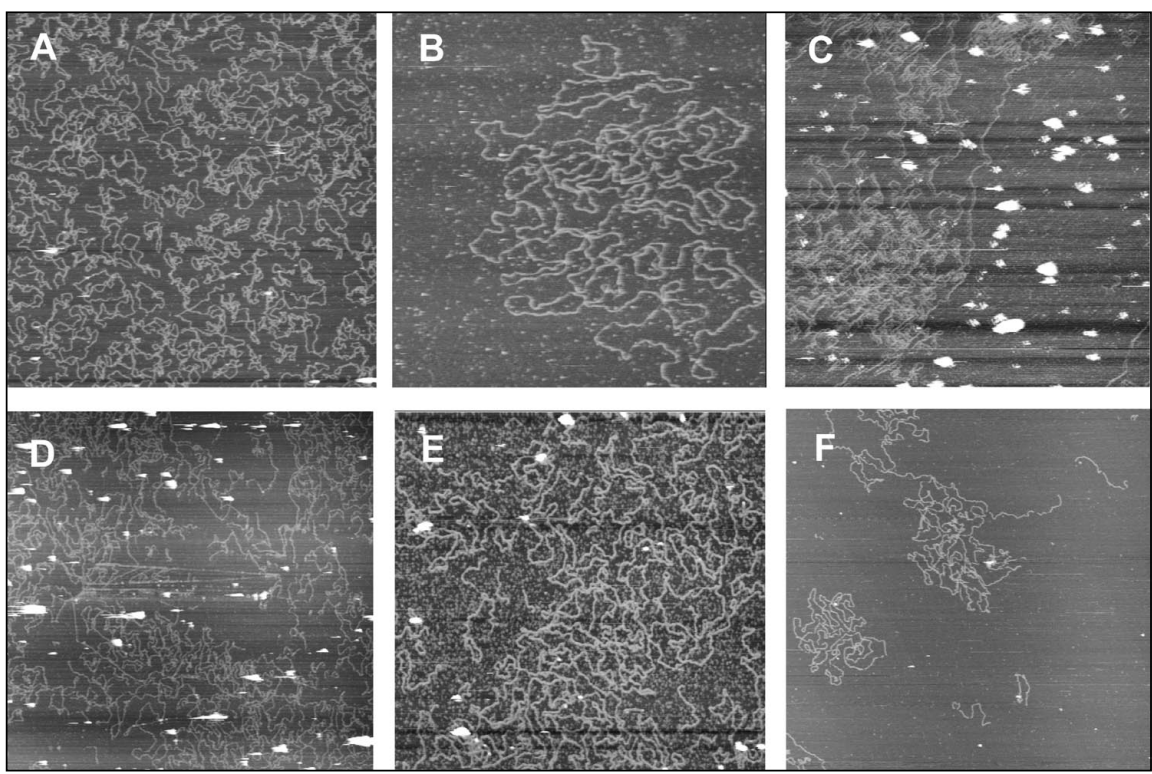

Figure 2. Analysis of plasmid and bacterial artificial chromosome (BAC) DNA preparations by atomic force microscopy (AFM). AFM was used to compare (A) a control plasmid DNA preparation to (B) BAC DNAs isolated by equilibrium sedimentation in $\mathrm{CsCl}$ or $(\mathrm{C}-\mathrm{E})$ various commercial BAC DNA maxi-preparation kits. Preparations of BAC DNA from commercial purification columns showed variable degrees of contamination with debris (white spots), which in most instances could be traced to dissociated column resin. The organic extraction and precipitation with ethanol of the preparation in panel $\mathrm{E}$ resulted in cleaner DNA but with a concomitant loss of DNA concentration and some (F) DNA breakage. Each field represents a 3.5-5.0 $\mu \mathrm{m}$ AFM scan. 


\section{Formation of Transfection Complexes}

We used AFM in biologically relevant buffers to explore the formation of BAC transfection complexes. In the absence of DNA, AFM showed that cationic lipids such as Lipofectamine 2000 formed micelles under ambient fluid conditions that ranged in diameter from 70 to $300 \mathrm{~nm}$ (Figure 3A). By varying the ratios of cationic lipids to DNA, conditions for AFM were defined that allowed us to examine the process of the formation of transfection complexes with different DNA preparations and transfection reagents. Using AFM, we observed partially formed but discrete plasmid DNA lipoplexes that ranged 80-500 nm (Figure 3B). When fully formed, these complexes displayed no free DNA and appeared to contain 1-10 plasmid molecules. Plasmid lipoplexes mediated the high-efficiency transfection of hamster $\mathrm{CHOC}$ 400 , human Saos-2, or mouse C10 cell lines under a broad range of conditions (data not shown). The condensation of plasmid DNA with the protein cofactor GST-Z2 or proprietary commercial condensation reagents had little or no effect on the transfection efficiency (data not shown).

The transfection of BACs depends on the formation of cationic lipid:DNA complexes that must both gain access to the cell and disassemble upon cell entry (16). In contrast to plasmid DNA, the formation of transfection complexes with BACs requires both the efficient condensation of individual BAC DNA molecules and packaging of the condensed DNA within a lipid shell. Using the same concentrations of BAC DNA as plasmid DNA, AFM showed that BAC complexes formed by condensation with the PLUS reagent (Figure 3C) or Lipofectamine (Figure 3D) were 10-100 times larger than plasmid DNA complexes and often contained unprotected DNA. These intermolecular aggregates did not mediate transfection. As previously demonstrated with BAC 269 (17), the condensation of B16-25 with GST-Z2 (Figure 3E) prior to incubation with Lipofectamine resulted
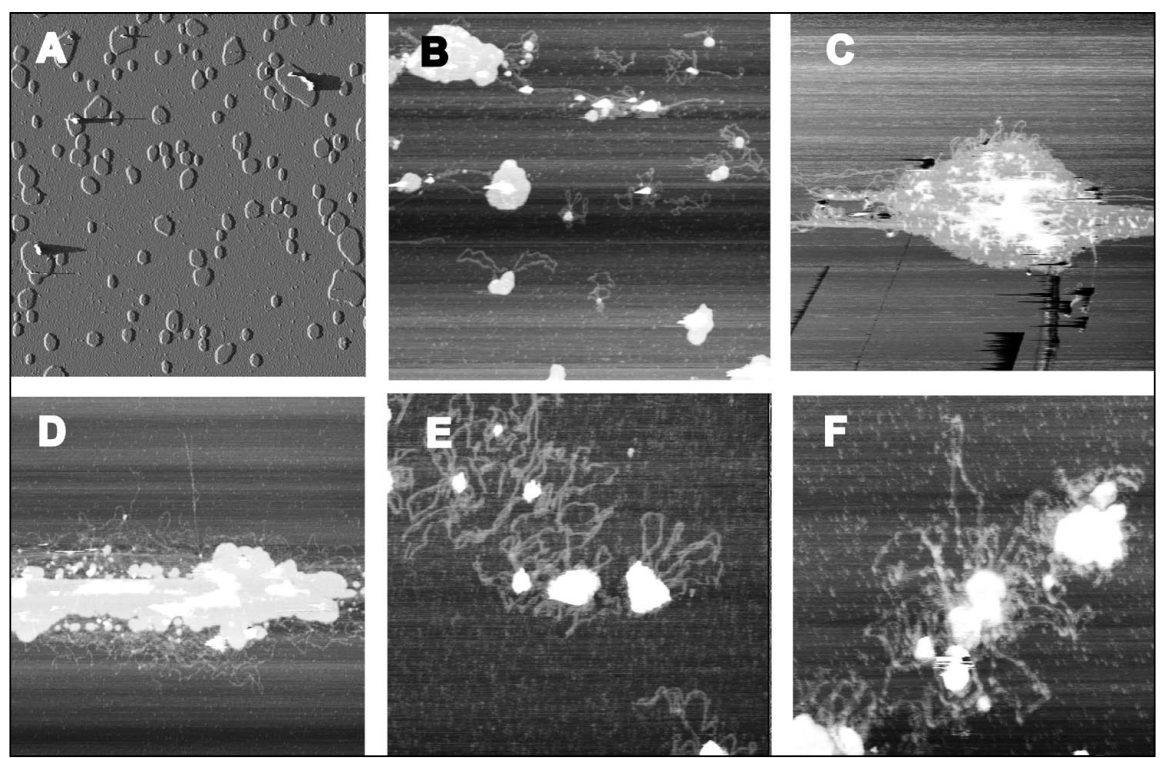

Figure 3. Analysis of plasmid and bacterial artificial chromosome (BAC) lipoplex formation by atomic force microscopy (AFM). The formation of lipoplexes containing plasmid DNA or BAC DNA with Lipofectamine, PLUS reagent, or Lipofectamine 2000 was examined by AFM. (A) Lipofectamine 2000 alone formed flattened micelles ranging in diameter from 70 to $300 \mathrm{~nm}$. (B) Lipofectamine 2000 induced the formation of a wide range of plasmid lipoplexes that appeared to contain 1-10 plasmid molecules. These conditions correspond to conditions that yield high-efficiency transfection of CHOC 400 and C10 cells, except that the final concentration of transfection reagent was lowered to permit AFM imaging. The incubation of BAC DNA with (C) PLUS reagent or (D) Lipofectamine, under conditions that mediate efficient plasmid transfection, resulted in large aggregates that did not mediate transfection. (E) Condensation with GST-Z2 promoted the formation of discrete protein-BAC DNA complexes, as previously reported (17). (F) By reducing the concentration of DNA and Lipofectamine 2000, discrete BAC DNA complexes that support transfection were observed. in better transfection rates in $\mathrm{CHOC}$ 400 cells (Figure 1B; data not shown). In contrast, Lipofectamine 2000 promoted the formation of discrete BAC lipoplexes (Figure 3F) comparable in size to plasmid lipoplexes, and these complexes supported transfection in C10 cells (Figure 1D; data not shown).

Plasmid preparations usually contain a high proportion of supercoiled molecules, whereas BAC DNA preparations are more heterogeneous, with a higher percentage of linear and relaxed circular DNA. Intermolecular linking of BAC DNA appears to be primarily a function of the size of the DNA molecules, which suggests that linear and relaxed circles may encourage the formation of DNA networks. It is possible that regions of larger BAC DNA molecules may remain free of lipid for a longer time in solution, promoting interactions between complexes, thereby causing the formation of large-linked multilamellar structures that are unsuitable for cell fusion $(12,26)$.

\section{Development of BAC Transfection Protocols for Saos-2 Cells}

Using AFM and the expression of EGFP from BAC B16-25 to track transfection, we developed conditions for BAC condensation with GST-Z2 and transfection with cationic lipids that approach $30 \%$ in CHOC 400 cells (17). We were also able to develop efficient transfection conditions for mouse $\mathrm{C} 10$ cells by manipulating the BAC DNA concentration, condensation conditions with PLUS reagent, and formation of discrete lipoplexes with Lipofectamine (Figure 1D). However, human osteosarcoma Saos- 2 cells displayed transfection efficiencies less than $0.1 \%$ with the GST-Z2 and PLUS reagent/Lipofectamine protocols (data not shown).

For Saos-2, we tested three commercial transfection reagents that do not require a DNA condensation step, presumably because they contain both fusigenic and cationic lipids. Based on conditions for lipoplex formation with Lipofectamine 2000 that resulted in discrete complexes (Figure 3F), we examined the relationships among BAC DNA concentration, Lipofectamine 2000:BAC DNA ratios, and transfection efficiency in Saos-2 cells. Two 
different concentrations of B16-25 BAC DNA were incubated with volumes of Lipofectamine 2000, and the reaction mixtures were used to transfect subconfluent Saos-2 cells. The cells were fixed $48 \mathrm{~h}$ after transfection, stained with DAPI, and examined by fluorescence microscopy for EGFP expression. At DNA:Lipofectamine 2000 ratios (expressed as $\mu \mathrm{g}$ DNA/ $\mu \mathrm{L}$ transfection reagent) of $1: 1$ and $1: 1.5$ (data not shown), significant numbers of transfected cells were clearly visible by $48 \mathrm{~h}$ (Figure 4A). At these DNA: Lipofectamine ratios, transfection complexes are barely visible by DAPI staining (Figure 4E). At higher DNA concentrations, which led to intermolecular linking and large aggregates as observed by AFM, transfection complexes were clearly visible by DAPI staining (Figure 4, G and $\mathrm{H}$ ). Although complexes of this size were found in association with individual cells, there was little evidence of EGFP expression in cells transfected under these conditions.

\section{Gene Expression after BAC Transfection}

The signal intensity and time of expression after transfection represent important parameters for assessing
BAC transfection efficiency. With B16-25 BAC transfections, we occasionally observe in all cell types a small percentage of very highly fluorescent EGFP-positive cells that do not persist after $24 \mathrm{~h}$ (data not shown). More often, cells transfected with BACs display very faint fluorescent signals by 24 $\mathrm{h}$, and cells with more visible signals are readily evident by 3 (Figure 5B) or 7 days (Figure 5C). Immunoblotting and immunofluorescence microscopy indicate that by 3-5 days, these cells express p53 (data not shown). In other experiments, immunoblotting shows that the expression of p53 from BACs in transient transfection experiments occurs at 100- to 1000-fold lower levels than for cells transfected with pCMV-p53 expression plasmids (Figure 1E; Reference 17).

Cells transfected with plasmids containing the same pCMV-EGFP expression cassette as B16-25 show high levels of fluorescence by $24 \mathrm{~h}$ (Figure 5D). However, by 7 days, very few cells transfected with pCMV-EGFP are evident, perhaps due to high levels of EGFP expression. The difference in EGFP signal intensity between plasmids and BACs is likely related to the copy number of the transfected DNA, but it is also possible that BACs replicate in culture because of their large

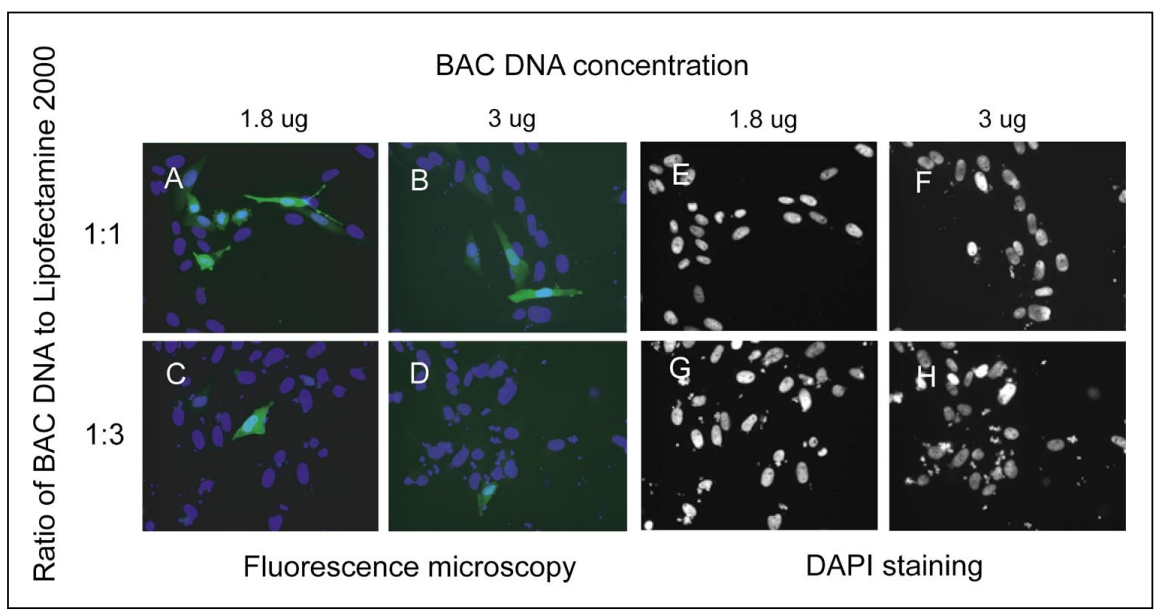

Figure 4. Transfection of Saos-2 cells with B16-25. Saos-2 cells were plated at approximately 50\% confluency and transfected with either (A-D) 1.8 or $3 \mu \mathrm{g}$ B16-25 bacterial artificial chromosome (BAC) DNA per well. Based on size, these concentrations of BAC DNA correspond to 0.075 and $0.125 \mu \mathrm{g}$, respectively, of a 5-kb pCMV-enhanced green fluorescent protein (EGFP) expression plasmid. The ratio of BAC DNA to transfection reagent ( $\mu \mathrm{g}$ DNA: $\mu \mathrm{L}$ reagent) varied from 1:1 to 1:3, as indicated. Two days after transfection, the cells were fixed, stained with 4',6'-diamidino-2-phenylindole (DAPI), and examined for EGFP expression by fluorescence microscopy (40× magnification). Processing of the images in panels E-H to accentuate the DAPI signal showed that high DNA concentrations and high ratios of transfection reagent to DNA resulted in $(\mathrm{G}$ and $\mathrm{H})$ large aggregates that were visible by fluorescence microscopy. 
size and therefore persist for longer times than do plasmids. In general, the examination of transfection by fluorescence microscopy or immunoblotting after 3-5 days invariably showed that a much larger fraction of cells was transfected with BACs than was anticipated from analyzing results after $24-48 \mathrm{~h}$, which is common with plasmid transfections. Finally, for reasons unknown, BAC transfections were uniformly 2-3 times more efficient with cells plated on plastic culture dishes than glass coverslips (data not shown).

\section{Co-Transfection of BAC Clones}

An important application in plasmid transfection is the ability to co-transfect multiple plasmids into a single cell. Therefore, we examined the possibility of co-transfecting BACs by transfecting CHOC 400 cells with B1625 and N2A1 at a 1:1 molar ratio using the PLUS reagent and Lipofectamine protocol. Figure 6 shows that approximately $50 \%$ of the transfected cells express both RFP and EGFP under these conditions, which provides a method for introducing multiple BACs into
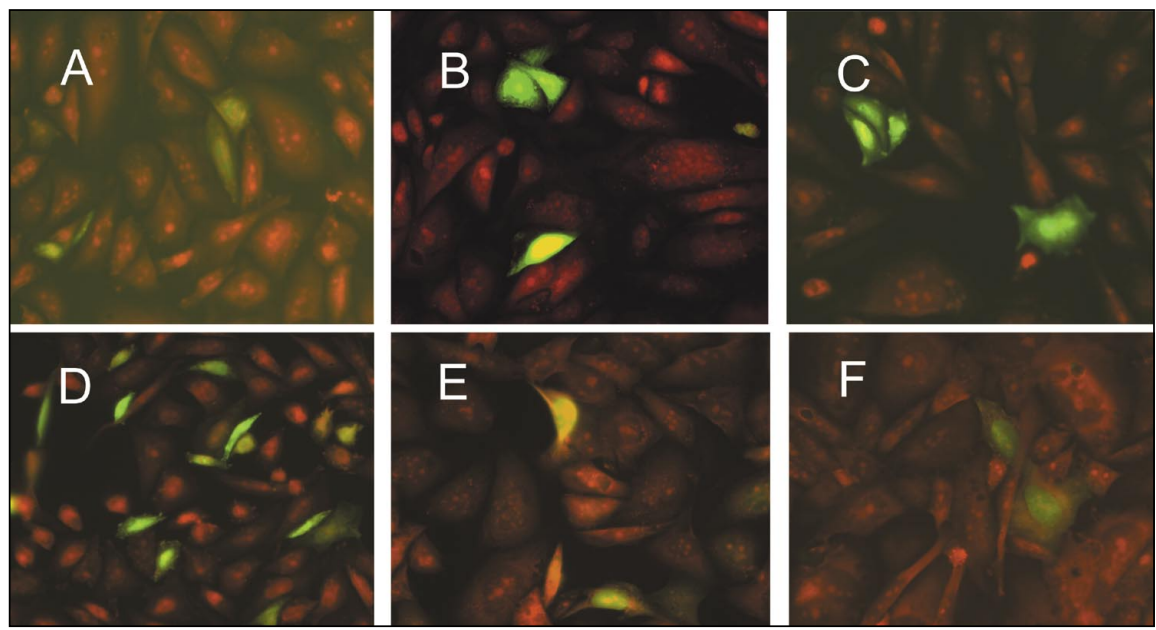

Figure 5. Delayed enhanced green fluorescent protein (EGFP) expression after transfection with bacterial artificial chromosomes (BACs). Chinese hamster ovary cells grown on glass coverslips were transfected with (A-C) B16-25 or (D-F) pCMV-EGFP. After (A and D) 1, (B and E) 3, or (C and F) 7 days, the cells were fixed and examined for EGFP expression by fluorescence microscopy. High levels of GFP expression are observed in (D) cells transfected with plasmids by $24 \mathrm{~h}$, whereas (A) cells transfected with B16-25 show a weak signal at this time point. (C) By 7 days, the cells transfected with BACs continue to express EGFP, whereas (F) few cells transfected with pCMV-EGFP express EGFP by this time.
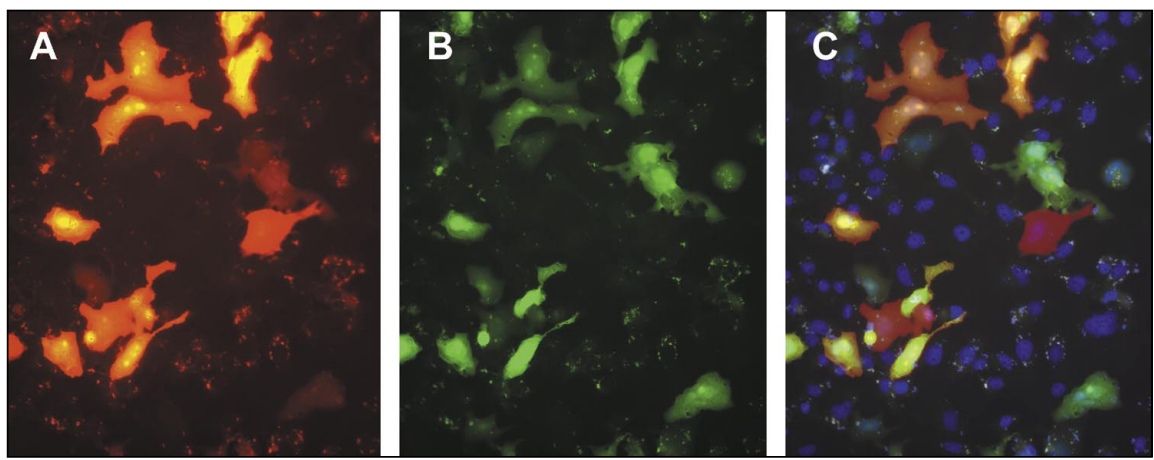

Figure 6. Co-transfection of bacterial artificial chromosomes (BACs) into CHOC 400 cells. BAC B16-25 and BAC N2A1 were mixed at a 1:1 molar ratio and transfected into CHOC 400 cells using a protocol optimized for PLUS reagent and Lipofectamine. After $72 \mathrm{~h}$, the cells were fixed, stained with $4^{\prime}, 6^{\prime}$-diamidino-2-phenylindole (DAPI), and examined by fluorescence microscopy ( $40 \times$ magnification) for the expression of (A) red fluorescent protein (RFP) or (B) enhanced green fluorescent protein (EGFP). (C) Merging the two images shows that approximately 50\% of the transfected cells are expressing both markers. Note that many cells associated with larger lipoplexes under these transfection conditions did not express RFP or EGFP. 
a single background. In addition, we have co-transfected pCMV plasmids that express gene-specific transcription factors such as E2F-1 with BAC clones, and here the number of transfected cells that received both plasmid and BAC DNA appeared to be $50 \%$ or higher (data not shown).

\section{DISCUSSION}

The introduction of foreign DNA into mammalian cells via transfection to analyze gene function and regulation is one of the most powerful tools available in cell and molecular biology. In the past, gene functions have been analyzed by the construction of expression vectors that link the cDNA copy of a gene to a powerful heterologous promoter, often of viral origin. Gene regulation studies also often involve linking relatively short promoter or enhancer regions to reporter genes and assaying for reporter gene expression. While these studies have provided valuable insights, it has become increasingly apparent that they are of limited use for examining the contributions of regulatory sequences that act over long distances to modify chromatin or DNA structure through DNA looping or linking. The ability to modify BAC (or P1 and YAC) clones in precise ways by homologous recombination allows for the functional dissection of regulatory elements in the context of entire genes and large amounts of flanking sequences, an approach that has proven successful in transgenic mouse models (3).

Transfection lipoplexes are thought to gain entry through the endosomal pathway $(13,15)$, but the mechanism of DNA escape from the complex, ejection from the endosome, survival in the cytoplasm, and transport to the nucleus is unknown. $\mathrm{Xu}$ and Szoka (16) proposed that the cationic lipoplex destabilizes the endosomal membrane, inducing anionic lipids to flip-flop from the cytoplasmic-facing monolayer. These lipids then form charge-neutral ion pairs with the cationic lipids, allowing the DNA to escape from the complex into the cytoplasm. The mechanism by which DNA gains access to the nucleus is unknown, but experimental evidence indicates that it must dissoci- ate from the cationic liposome before nuclear entry (33). For both plasmids and BACs, the generation of discrete complexes of $80-400 \mathrm{~nm}$ appears to be critical for efficient transfection.

The ability of the AFM to probe single molecules or complexes rather than examining a Gaussian distribution of them in physiologically relevant conditions allows for a unique form of molecular analysis. Subtle characteristics of DNA preparations such as the level of supercoiling, degree of strand breakage, or the presence of contaminants can be readily assessed. Lipoplex structures can be examined for the size, structure, and distribution of complexes, numbers of DNA molecules per complex, and level of exposure of DNA strands. All of these characteristics play important roles in the generation of high-efficiency transfection complexes and can be carefully monitored at multiple time points by AFM in the fluid-tapping mode $(34,35)$.
We have noted three general parameters that influence the efficiency of BAC transfection: (i) DNA purity and concentration; (ii) conditions that promote intramolecular condensation versus intermolecular linking; and (iii) cell type. The most common problem we have encountered is the contamination of BAC DNA preparations from commercial purification columns with debris, which varied considerably from kit to kit and even from column to column in the same kit. The contamination of DNA preparations had little or no effect on $\mathrm{A}_{260} / \mathrm{A}_{280}$ ratios or use of DNA for restriction endonuclease digestion, PCR, or electrophoresis. Although AFM indicated that debris from purification columns is incorporated into BAC transfection complexes where it may exert toxic effects upon cell entry, column debris appeared to inhibit transfection more directly by promoting the formation of large DNA-lipid 
aggregates through intermolecular linking. Although careful extraction with phenol:chloroform solutions can alleviate this problem (36), DNA purification by equilibrium sedimentation in $\mathrm{CsCl}$ is recommended for optimizing BAC transfection conditions. Once DNA recovered from purification columns is compared to a $\mathrm{CsCl}$ standard, the timesavings and increase in DNA recovery warrant the use of commercial kits.

The formation of discrete transfection complexes (as in Figure 3F) required conditions that promote intramolecular condensation. AFM indicates that intramolecular condensation by GST-Z2, PLUS reagent, or other commercial formulations is a function of BAC DNA size, DNA purity, DNA concentration, and the length of time of the condensation reaction. Increasing the DNA concentration or time of the condensation reaction almost invariably reduced transfection rates. Therefore, to optimize protocols for BAC transfection, we recommend that the initial focus be placed on titrating DNA concentrations downward from $0.5 \mu \mathrm{g} / \mu \mathrm{L}$ in the condensation reaction until one can observe small, well-dispersed complexes that can be evaluated by staining cell cultures with DAPI 48 $\mathrm{h}$ after transfection (Figure 4 ).

The examination of protein expression and EGFP or RFP fluorescence indicates that care must be taken in evaluating BAC transfection results. The analysis of stably transfected cells indicates that BACs persist at copy numbers from 5 to 20 per cell (S. Illenye and N.H. Heintz, unpublished observations). After the transfection of CHOC 400 cells grown on plastic at efficiencies nears $50 \%$, immunoblotting and immunofluorescence experiments show that EGFP expression is observed in some cells are early as $24 \mathrm{~h}$, but at least $72 \mathrm{~h}$ are required to detect the expression of p53 from $66 \mathrm{H} 22$ or B16-25 (Figures 1 and 5). After 3-7 days, numerous cells that express both EGFP and p53 emerge, which suggests that expression is improved once transfected cells have undergone one or more cell divisions.

Finally, although we generally have found that any cell that can be transfected with plasmids can be transfect- ed with BACs, there are significant differences in transfection efficiency from cell type to cell type. Sideby-side transfections with identical GST-Z2/Lipofectamine transfection mixtures showed more than a 100fold difference in efficiency between CHOC 400 and Saos-2 cells under the same culture conditions (data not shown). However, by the empirical testing of reagents and conditions that promote intramolecular condensation and varying the ratio between $\mathrm{BAC}$ DNA and the transfection reagent, we have found it possible to transfect BACs at reasonable levels in human, mouse, and hamster cell lines. Once a protocol is optimized for a specific cell type, co-transfection of two or more BACs is feasible by including equimolar amounts of each clone in the transfection mixture.

\section{ACKNOWLEDGMENTS}

We thank T. Quinn and D. Taatjes for assistance with AFM and cell imaging, X.W. Yang and N. Heintz for modification vectors, N. Nowak for assistance with BAC clones, the Vermont Cancer Center and DNA Analysis Facility, the Department of Pathology, and a grant (PO1 H0670004) from the National Heart Lung and Blood Institute (Washington, DC, USA) for research support. W.J.M. was supported by a grant (T32 ES07122) from the National Institute of Environmental Health Sciences (Research Triangle Park, NC, USA).

\section{REFERENCES}

1.Antoch, M.P., E.J. Song, A.M. Chang, M.H. Vitaterna, Y. Zhao, L.D. Wilsbacher, A.M. Sangoram, D.P. King, et al. 1997. Functional identification of the mouse circadian clock gene by transgenic BAC rescue. Cell 89:655-667.

2.Kaufman, R.M., C.T. Pham, and T.J. Ley. 1999. Transgenic analysis of a $100-\mathrm{kb}$ human beta-globin cluster containing DNA fragment propagated as a bacterial artificial chromosome. Blood 94:3178-3184.

3.Heintz, N. 2001. BAC to the future: the use of BAC transgenic mice for neuroscience research. Nat. Rev. Neurosci. 2:861-870.

4.John, R.M., S.A. Aparicio, J.F. Ainscough, K.L. Arney, S. Khosla, K. Hawker, K.J Hilton, S.C. Barton, et al. 2001. Imprinted expression of neuronatin from modified BAC transgenes reveals regulation by distinct and 
distant enhancers. Dev. Biol. 236:387-399.

5.Yang, X.W., P. Model, and N. Heintz. 1997. Homologous recombination based modification in Escherichia coli and germline transmission in transgenic mice of a bacterial artificial chromosome. Nat. Biotechnol. 15: 859-865.

6.Muyrers, J.P., Y. Zhang, G. Testa, and A.F. Stewart. 1999. Rapid modification of bacterial artificial chromosomes by ET-recombination. Nucleic Acids Res. 15:1555-1557.

7.Nefedov, M., R. Williamson, and P.A. Ioannou. 2000. Insertion of disease-causing mutations in BACs by homologous recombination in Escherichia coli. Nucleic Acids Res. 1:E79.

8.Yu, D., H.M. Ellis, E.C. Lee, N.A. Jenkins, N.G. Copeland, and D.L. Court. 2000. An efficient recombination system for chromosome engineering in Escherichia coli. Proc. Natl. Acad. Sci. USA 97:5978-5983.

9.Felgner, P.L., Y. Barenholz, J.P. Behr, S.H. Cheng, P. Cullis, L. Huang, J.A. Jessee, L. Seymour, et al. 1987. Lipofection: a highly efficient lipid-mediated DNA-transfection procedure. Proc. Natl. Acad. Sci. USA 84: 7413-7417.

10.Felgner, J.H., R. Kumar, C.N. Sridar, C.J. Wheeler, Y.J. Tsai, R. Border, P. Ramsey, M. Martinet al. 1994. Enhanced gene delivery and mechanism studies with a novel series of cationic lipid formulations. J. Biol. Chem. 269:2550-2561.

11.Farhood, H., N. Serbina, and L. Huang. 1995. The role of dioleoyl phosphatidylethanolomine in cationic liposome mediated gene transfer. Biochim. Biophys. Acta 1235: 289-295.

12.Oberle, V., U. Bakowsky, I.S. Zuhorn, and D. Hoekstra. 2000. Lipoplex formation under equilibrium conditions reveals a threestep mechanism. Biophys. J. 79:1447-1454.

13. Wrobel, I. and D. Collins. 1995. Fusion of cationic liposomes with mammalian cells occurs after endocytosis. Biochem. Biophys. Acta 1235:296-304.

14.Gao, X. and L. Huang. 1995. Cationic liposome-mediated gene transfer. Gene Ther. 2: 710-722.

15.Friend, D.S., D. Papahadjopoulos, and R.J. Debs. 1996. Endocytosis and intracellular processing accompanying transfection mediated by cationic liposomes. Biochim. Biophys. Acta 1278:41-50.

16.Xu, Y. and F.C. Szoka. 1996. Mechanism of DNA release from cationic liposome/DNA complexes used in cell transfection. Biochemistry 35:5616-5623.

17.Montigny, W.J., C.R. Houchens, S. Illenye, J.M. Gilbert, E. Coonrod, Y-C. Chang, and N.H. Heintz. 2001. Condensation by DNA looping facilitates transfer of large DNA molecules into mammalian cells. Nucleic Acids Res. 29:1982-1988.

18.Hansma, P.K., J.P. Cleveland, M. Radmacher, D.A. Walters, P. Hillner, M. Benzanilla, M. Fritz, D. Vie, et al. 1994. Tapping mode atomic force microscopy in liquids. Appl. Phys. Lett. 64:1738-1740.

19.Putman, C.A., K.O. Van der Werf, B.G. De Grooth, N.F. Van Hulst, and J. Greve. 1994. Tapping mode atomic force micros- copy in liquid. J. Appl. Phys. 80:3622-3627.

20.Kasas, S., N.H. Thompson, B.L. Smith, H.G. Hansma, X. Zhu, M. Guthold, C. Bustamante, E.T. Kool, et al. 1997. Escherichia coli RNA polymerase activity observed using atomic force microscopy. Biochemistry 36:461-468.

21.Milbrandt, J.D., J.C. Azizkhan, and J.L. Hamlin. 1983. Amplification of a cloned hamster dihydrofolate reductase gene after transfer into a dihydrofolate reductase-deficient cell line. Mol. Cell. Biol. 3:1274-1282.

22.Reiners, J.J., A. Pavone, A.R. Cantu, C. Auerbach, and A.M. Malkinson. 1992. Differential expression of cytochrome P-450 in proliferating and quiescent cultures of murine lung epithelial cells. Biochem. Biophys. Res. Commun. 183:193-198.

23.Houchens, C.R., W.J. Montigny, L. Zeltser, L. Dailey, J.M. Gilbert, and N.H. Heintz. 2000. The $d h f r$ ori $\beta$-binding protein RIP60 contains 15 zinc fingers: DNA binding and looping by the central three fingers and an associated proline-rich region. Nucleic Acids Res. 28:570-581.

24.Chang, Y.C., S. Illenye, and N.H. Heintz. 2001. Cooperation of E2F/p130 and Sp1/ $\mathrm{pRb}$ complexes in repression of the Chinese hamster dhfr gene. Mol. Cell. Biol. 21:11211131.

25.Xu, Y., S-W. Hui, P. Frederik, and F.C. Szoka. 1999. Physicochemical characterization and purification of cationic lipoplexes. Biophys. J. 77:341-353.

26.Zhang, Y.P., L. Sekirov, E.G. Saravolac, J.J. Wheeler, P. Tardi, K. Clow, E. Leng, R. Sun, et al. 1999. Stabilized plasmed-lipid particles for regional gene therapy: formulation and transfection properties. Gene Ther. 6:1438-1447.

27.Lasic, D.D., H. Strey, M.C. Stuart, R. Podgornik, and P.M. Frederik. 1997. The structure of DNA-liposome complexes. J. Am. Chem. Soc. 119:832-833.

28.Radler, J.O., I. Koltover, T. Salditt, and C.R. Safinya. 1997. Structure of DNA-cationic liposome complexes: DNA intercalation in multilamelar membranes in distinct interhelical packing regimes. Science 275: 810-814.

29.Marschall, P., N. Malik, and Z. Larin. 1999. Transfer of YACs up to $2.3 \mathrm{Mb}$ intact into human cells with polyethylenimine. Gene Ther. 6:1634-1637.

30.Hansma, H.G., R. Golan, W. Hsieh, C.P. Lollo, P. Mullen-Ley, and D. Kwoh. 1998. DNA condensation for gene therapy as monitored by atomic force microscopy. Nucleic Acids Res. 26:2481-2487.

31.Dunlap, D.D., A. Maggi, M.R. Soria, and L. Monaco. 1997. Nanoscopic structure of DNA condensed for gene delivery. Nucleic Acids Res. 25:3095-3101.

32.Shizuya, H., B. Birren, U.J. Kim, V. Mancino, T. Slepak, Y. Tachiiri, and M. Simon. 1992. Cloning and stable maintenance of 300-kilobase-pair fragments of human DNA in Escherichia coli using an F-factorbased vector. Proc. Natl. Acad. Sci. USA 89: 8794-8797.

33.Zabner, J., A.J. Fasbender, T. Moninger, K.A. Poellinger, and M.J. Welsh. 1995.
Cellular and molecular barriers to gene transfer by a cationic lipid. J. Biol. Chem. 270:18997-19007.

34.Bustamante, C. and C. Rivetti. 1996. Visualizing protein-nucleic acid interactions on a large scale with the scanning force microscope. Annu. Rev. Biophys. Biomol. Struct. 25:395-429.

35.Engel, A., Y. Lyubchenko, and D. Muller. 1999. Atomic force microscopy: a powerful tool to observe biomolecules at work. Trends Cell Biol. 9:77-80

36.Gama Sosa, M., R. De Gasperei, P. Wen, E. Gonzalez, K. Kelley, R. Lazzarini, and G. Elder. 2002. BAC and PAC DNA for the generation of transgenic animals. BioTechniques 33:51-53.

Received 10 February 2003; accepted 17 June 2003.

Address correspondence to:

Nicholas H. Heintz

Department of Pathology

University of Vermont College of Medicine

89 Beaumont Avenue

Burlington, VT 05405, USA

e-mail: nicholas.heintz@uvm.edu 